\title{
Postpartum laparoscopic sterilisation: A role in South African healthcare?
}

\author{
D L Prince, ${ }^{1} \mathrm{MB}$ ChB, Dip Obst (SA); R M Aronius, ${ }^{2} \mathrm{MB}$ ChB, MMed O\&G; \\ G B Theron, ${ }^{1} \mathrm{MB}$ ChB, FCOG (SA), MMed (O\&G), BSc Hons, MD \\ ${ }^{1}$ Department of Obstetrics and Gynaecology, Stellenbosch University and Tygerberg Hospital, Cape Town, South Africa \\ ${ }^{2}$ Department of Obstetrics and Gynaecology, Worcester Hospital, Worcester, South Africa
}

Corresponding author: D L Prince (deborahlynneprince@gmail.com)

Objective. To assess the advantages and feasibility of performing postpartum sterilisations laparoscopically in a public healthcare facility in South Africa (SA).

Methods. Retrospective review of postpartum sterilisations between June 2012 and December 2013 at Worcester Hospital, Western Cape, SA. A total of 78 postpartum sterilisations were included in the study (open $n=26$, laparoscopic $n=52$ ). Data analysis was performed using means and medians with range and standard deviations, two-sample Wilcoxon rank sum test, two-sample $t$-test and $\chi^{2}$ test.

Results. It was considered preferable to perform laparoscopic sterilisations than the open procedure on patients with a higher body mass index (BMI). Duration of surgery was shorter in the laparoscopic group, especially in patients with BMI $>30$, and more patients were discharged on the same day as surgery in the laparoscopic group. There were fewer overall complications in the laparoscopic group (odds ratio $0.35,95 \%$ confidence interval $0.08-1.43$ ).

Conclusion. It is feasible to perform postpartum sterilisations laparoscopically in a public healthcare facility in SA. Advantages of the procedure are clinically significant and in keeping with international literature. Future research should be undertaken regarding acceptability of the procedure in the study population, laparoscopic training and the improvement of provision of requested postpartum sterilisations in public hospitals.

S Afr J Obstet Gynaecol 2016;22(1):4-7. DOI:10.7196.SAJOG.2016.v22i1.1019

The need for the introduction of laparoscopic postpartum sterilisations in the public healthcare sector in South Africa (SA) has been identified through the weaknesses found in the current practice of performing all postpartum sterilisations (PPS) as open procedures. The international literature contains many reports of the advantages of doing the procedure laparoscopically, and the feasibility of the procedure, although once disputed, is widely accepted. ${ }^{[1-3]}$ The aim of our study was primarily to prove feasibility and assess advantages of postpartum laparoscopic sterilisation in a public healthcare facility in SA, as a developing country. Our secondary aims were to assess the ease of the procedure and complication rates, to illustrate the procedure's proposed impact on hospital stay and operating time and to ascertain the potential of the procedure to increase postpartum sterilisation rates in the future.

\section{Methods}

The study is a retrospective descriptive study and reviews all clinical records of patients who underwent both open and laparoscopic postpartum sterilisations at Worcester Hospital, a secondary level hospital in the Western Cape Province, SA, between June 2012 and December 2013.

Postpartum sterilisation is defined as a sterilisation performed within 72 hours of the vaginal delivery of a viable baby. (In our setting viability is defined as 28 weeks or $1000 \mathrm{~g}$ ). Excluded from the study were sterilisations performed more than 72 hours after vaginal delivery, any sterilisation performed after the delivery of a non-viable fetus and any sterilisation performed concurrently with any other procedure (e.g. caesarean section, perineal tear repair).

Laparoscopic and open sterilisations were performed under local, regional or general anaesthesia as decided by the anaesthetist involved in the case. Laparoscopic sterilisations were performed with a FalopeRing applicator/bipolar grasper and open sterilisations by means of the modified Parklands method. Laparoscopic sterilisations were all performed with two ports, an upper umbilical abdominal entry site with either a $10 \mathrm{~mm}$ or $5 \mathrm{~mm}$ port for a $30^{\circ}$ lens and a $7 \mathrm{~mm}$ suprapubic port for the Falope-Ring applicator. Insufflation was with a Veress needle if the patient had no prior midline surgery and intraabdominal adhesions were not suspected, and otherwise with an open Hasson technique or insufflation at Palmer's point. After completion of the sterilisation the sheath was closed with the $10 \mathrm{~mm}$ ports and skin closure was with polyglactin dissolvable sutures.

The open procedure was performed with a $2-4 \mathrm{~cm}$ sub-umbilical incision using the modified Parklands method, which includes the ligation and excision of a portion of the fallopian tube. The sheath was closed with absorbable sutures and the skin with absorbable sutures or skin clips. Duration of surgery was defined from first incision to skin closure.

Patient information was obtained though the theatre and labour ward registers, as well as a computer database of all surgeries performed at Worcester Hospital. Folders of these patients were then requested, and the hardcopy folder was audited according to certain defined parameters and information recorded on an anonymous data capture sheet. Patients with incomplete records, interval procedures or non-viable deliveries were excluded. Information from the data capture sheets was entered into an Excel spreadsheet (Microsoft Corp., USA) for analysis.

Data analysis was performed using means and standard deviations (SDs) and the two-sample $t$-test for normally distributed data. The median and range with the two-sample Wilcoxon rank-sum test was used with data not normally distributed. The $\chi^{2}$ test without Yates' correction was used for qualitative data analysis. 
There was no need for informed consent for the study because it was a retrospective review and no funding was required.

Ethical approval was obtained from the Stellenbosch University Health Research Ethics Committee (S13/08/139). Additional approval was obtained from the chief executive officer of Worcester Hospital and the Western Cape Department of Health.

\section{Results}

During the study period between July 2012 and December 2013, 425 sterilisations were performed at Worcester Hospital. Excluded from the study were sterilisations performed with caesarean section $(n=217)$, sterilisations performed in conjunction with other procedures $(n=8)$, interval sterilisations $(n=57)$ and sterilisations with missing folders $(n=47)$. Of the 96 postpartum sterilisations performed, 18 were excluded because of incomplete notes, 26 sterilisations were performed open via mini-laparotomy and 52 were performed laparoscopically.

The main outcome measures were chosen to assess the feasibility and possible advantages of this procedure in a public hospital in SA. The demographic data were chosen to describe the patients in whom this procedure is practical, including patients with an increased body mass index (BMI).

One main outcome was duration of surgery of sterilisations performed via mini-laparotomy compared with the laparoscopic procedure. This outcome was divided into three groups: the first group assessed the time difference between all open v. laparoscopic procedures; the second group assessed time differences in duration of surgery in laparoscopic v. open procedures among patients with a $\mathrm{BMI} \geq 30$; and the third group compared duration of surgery of laparoscopic v. open procedures performed by surgeons with intermediate experience (community service doctors, medical officers and registrars). Outcomes also included the time the patient had to wait post delivery before the sterilisation procedure (hours) and the length of stay postoperatively (days).

The total complications experienced in open v. laparoscopic sterilisations were recorded. These complications were divided into complications occurring during surgery, early complications (before discharge) and late complications (after discharge, excluding failed sterilisations).

Laparoscopic sterilisations were all performed under general anaesthesia with a Falope-Ring applicator. All but one of the open procedures were performed under general anaesthesia (one case spinal anaesthesia). All open procedures were performed through a sub-umbilical incision and the sterilisation was performed using the modified Parklands method. There were seven patients with previous abdominal surgery in the postpartum laparoscopic group. Only one patient had a non-umbilical (Palmer's point) entry site and this was due to a previous midline incision. The six patients with previous Pfannenstiel incisions had an uncomplicated entry through an umbilical incision. In all laparoscopic cases insufflation was with the use of a Veress needle.

All patients had a single consent form from the Provincial Administration of the Cape of Good Hope, including a checklist.

Demographics were similar between the two groups, with mean age and parity being almost identical (Table 1). On average the laparoscopic group had a slightly higher BMI with a maximum BMI of 46 compared with the maximum BMI in the open group of 38.8. (The maximum BMI managed at Worcester Hospital is 49 because of anaesthetic risk.)
Total duration of surgery was found to be 3.7 minutes shorter in the laparoscopic than the open group (95\% confidence interval (CI) $-1.1-8.5 ; p=0.08$ ) (Table 2). Duration of surgery in the subgroup of patients with a BMI $>30$ was 4.9 minutes shorter in the laparoscopic group $(n=21)$ compared with the open group $(n=6)$ (95\% CI -5.3 - 15.2; $p=0.83$ ). Lastly, comparing duration of surgery within the subgroup of intermediate surgeons revealed that the open procedure $(n=22)$ was 0.65 minutes shorter than the laparoscopic procedure $(n=21)(95 \%$ CI $-7.3-6.0 ; p=0.81)$.

Average waiting time post delivery for the sterilisation procedure was 13.5 hours in the open group and 17.7 hours in the laparoscopic group (Table 3). Length of stay in hospital was comparable between the two groups but more laparoscopic patients were discharged on the same day as the procedure.

Total complications were found to be fewer in the laparoscopic group (odds ratio (OR) 0.35, 95\% CI 0.08 - 1.43; $p=0.14$ ) (Table 4). Complications experienced during surgery in the open group included bleeding from the muscle and sheath $(n=1)$, suture slipped with bleeding $(n=1)$, and difficulty finding tube $(n=1)$. In the laparoscopic group the three complications during surgery were

Table 1. Demographic data of patients included in the study

\begin{tabular}{llll}
\hline Characteristic & Open & Laparoscopic & $\boldsymbol{p}$-value \\
\hline Age (years)* & $33.9(5.4)$ & $33.9(5.2)$ & 0.77 \\
BMI* $^{*}$ & $26.2(5.6)$ & $28.3(6.9)$ & 0.21 \\
Maximum BMI & 38.8 & 46 & \\
Parity & \\
*Data reported as mean (SD). & $4.5(1.2)$ & $4.3(1.0)$ & 0.44
\end{tabular}

Table 2. Duration of surgery

\begin{tabular}{llll}
\hline & Total & BMI > 30 & Intermediate surgeons \\
\hline Open $^{*}$ & $25.0(9.52)$ & $29.5(4.4)$ & $24.8(1.8)$ \\
Laparoscopic & $21.3(10.2)$ & $24.5(2.3)$ & $25.4(2.7)$ \\
Difference & 3.65 & 4.9 & -0.65 \\
95\% CI & $-1.1-8.5$ & $-5.3-15.2$ & $-7.3-6.0$ \\
p-value & 0.08 & 0.83 & 0.81 \\
*Data reported as mean (SD). & & \\
\end{tabular}

Table 3. Waiting time for surgery and discharge

\begin{tabular}{lll}
\hline & Laparoscopic & Open \\
\hline $\begin{array}{l}\text { Average time post partum (hours), mean (SD) } \\
\text { Average length of stay post surgery (days), }\end{array}$ & $17.7(8.4)$ & $13.5(9.4)$ \\
mean (range) & & $1.52(1-3)$
\end{tabular}

Table 4. Complications encountered with postpartum sterilisations ${ }^{\star}$

\begin{tabular}{lll}
\hline & Laparoscopic & Open \\
\hline Complications in surgery, $n$ & 3 & 3 \\
Early complications, $n$ & 1 & 2 \\
Late complications, $n$ & 0 & 0 \\
Total surgery-related complications (\%) & 7.6 & 19.2 \\
${ }^{*}$ OR $0.35,95 \%$ CI $0.08-1.43$ & &
\end{tabular}


instrument failure $(n=2)$ and transection of the tube with bleeding that was stopped by a bipolar grasper without converting to an open procedure $(n=1)$.

Early complications (that occurred before discharge of the patient) included two complications in the open group consisting of postoperative pyrexia of unknown origin and a wound haematoma that was treated conservatively. In the laparoscopic group, one early complication occurred involving wound dehiscence of a $10 \mathrm{~mm}$ umbilical incision needing closure in theatre. No late complications were reported in either group.

A total of six complications (out of nine) were associated with the group of intermediate surgeons; five of these were during an open procedure and one during a laparoscopic sterilisation.

\section{Discussion}

SA has an unintended pregnancy rate of 59\% and a teenage pregnancy rate of $4.9 \% .{ }^{[4]}$ There is a $25 \%$ unmet need for family planning in women who are married or in a union. ${ }^{[5]}$ There is a large problem with loss to followup, especially in young women who are/have recently been pregnant. ${ }^{[6]}$ Women over the age of 15 who are clinically obese (BMI $\geq 30$ ) number $12.5 \%$, with a $35.7 \%$ prevalence of obesity among urban African women. ${ }^{[7]}$ Attitudes towards sterilisation may be influenced by cultural beliefs about fertility, accessibility to services and the sociopolitical history of SA during and after apartheid. ${ }^{[8]}$ Negative influences on the attitude towards sterilisation could also include failure to obtain requested sterilisation (most often due to physician dissuasion) $)^{[9,10]}$ and the stigma surrounding the forced sterilisation of HIVpositive women. ${ }^{[1]}$

There is much international literature describing the advantages of laparoscopic methods for sterilisation over an open procedure resulting in fewer instances of minor operative morbidity, shorter duration of surgery, minimal scarring and less postoperative discomfort. ${ }^{[1,2]}$ This was confirmed by the results of this study with up to 5 minutes' shorter duration of surgery (especially in the high-BMI group) and fewer overall complications (OR 0.35). A small case review by Garcia-Padial et al..$^{[12]}$ looking at laparoscopic postpartum sterilisation in women with a BMI $>35$ shows that the laparoscopic procedure is faster than an open procedure in women of similar weights with no reported complications. This proves that this procedure is safe and effective in the increasing proportion of women in SA with BMIs over 30. A study by Huber et al. ${ }^{[3]}$ that reviewed 27653 women undergoing sterilisation in Switzerland concluded that laparoscopic sterilisation, whether performed as an interval or postpartum procedure, is superior over a postpartum mini-laparotomy in terms of major and minor complications.

An interesting secondary outcome of our study is that intermediate surgeons (community service doctors/medical officers/registrars) with little or no previous laparoscopic experience took almost the same length of time to perform the laparoscopic as the open procedures, with fewer complications. In many countries it is mostly experienced surgeons who perform laparoscopic work, but in SA, however, the intermediate surgeons drive the maternal service and are responsible for almost all sterilisations performed. The authors were unable to adequately assess the learning curve of these doctors as numbers of sterilisations performed by each was too few, but this may be a topic for future research.

A topic of controversy surrounding this procedure is that of sterilisation method and sterilisation failure. It has been widely taught that sterilisations performed during the puerperium have an increased failure rate. Although there are studies showing an increase in failure rates when sterilisation is performed post partum, ${ }^{[13]}$ there are often many confounding factors, including surgeon experience, method used and follow-up. There are also studies which show no association between timing of sterilisation and failure, ${ }^{[14]}$ and the US Collaborative Review of Sterilisation, the longest cumulative outcome study on various sterilisation methods, found that the postpartum partial salpingectomy, the only method being performed post partum, had the lowest cumulative probability of sterilisation failure compared with other interval methods. ${ }^{[15]}$ Factors associated with an increased risk of sterilisation failure that were consistent were lower age and less surgeon experience. ${ }^{[13-15]}$

Methods of laparoscopic sterilisation include electrocoagulation, silicone band and clip being the most common. Mechanical devices have traditionally been preferred to electrocoagulation owing to risk of bowel injury with unipolar coagulation and poor outcomes of sterilisation reversal as a result of the destruction of tissue. ${ }^{[16]}$ Both the Filshie clip and Falope/Yoon ring have had varied studies of varying sizes performed, with varying follow-up intervals to assess their efficacies. ${ }^{[13-17]}$ As expected, results on failure rates are extremely varied and are influenced by surgeon experience, technique used, age of patient and duration of followup. ${ }^{[18]}$ It is therefore difficult for these authors to say with any certainty that one method is superior to the other, especially in a postpartum situation. Sterilisation failure was not a primary outcome in our study as it was a retrospective review with no confirmatory imaging (e.g. hysterosalpingogram) or followup interviews. A long-term cumulative outcome study is needed on different methods of laparoscopic postpartum sterilisation.

Suggested surgical techniques resulting from experience gained by the authors during the course of the study include:

- The surgeon must ensure that the instruments are functional before the administration of anaesthetic or first incision made.

- A blade facing upwards will prevent a skin incision that is too deep on entry.

- An upper umbilical site for abdominal entry is usually uncomplicated and performed easily in a postpartum patient. There may be instances, however, where a supra-umbilical or non-umbilical site (Palmer's point) is preferable, e.g. previous pelvic surgery.

- Retracting the fallopian tube slowly into the applicator will reduce the incidence of tubal transection.

- The round ligament, tube and fimbrial ends must be reviewed before closure to avoid the chance of operator-related sterilisation failure.

Lastly, we have found that it is practical to keep the laparoscopic set simple and small with reusable instruments. The instruments that were used are detailed in Table 5.

Some recommendations for future research would include looking at the

Table 5. Instruments used in laparoscopic procedure

$5 \mathrm{~mm}$ lens with $30^{\circ}$ angle

$5 \mathrm{~mm}$ reusable port (for the lens)

$7 \mathrm{~mm}$ reusable port for applicator

Falope-Ring applicator and rings

Size 11 scalpel

Stitch scissors and forceps

A disposable or reusable Veress needle may be included 
acceptability of the laparoscopic procedure among the SA patient population, the long-term follow-up of sterilisation failure from this procedure, an assessment of anaesthetic options to provide an ambulatory service and a quality improvement study to assess ways of improving the provision of requested postpartum sterilisations.

Strengths of the study were that it was a review of procedures performed in the population for which we are assessing its advantages. It is applicable to the limitations of the SA health service and the limited experience of surgeons in laparoscopic surgery, particularly in the public sector.

A limitation of this study is that it is a retrospective review of clinical records and was limited by the quality of note-keeping of the cases studied. Because it was retrospective, there was no randomisation of patients and therefore patients might have undergone selection bias as to which procedure was performed. Confounding factors may include surgeon experience, time of delivery and BMI.

\section{Conclusion}

It is feasible, safe and advantageous to provide a laparoscopic postpartum sterilisation service in SA. The service would allow patients to experience a procedure that has multiple advantages over open surgery, especially in a population such as in SA with a high loss to follow-up for interval procedures and changing demographics such as BMI.

\section{References}

1. Kulier R, Boulvain M, Walker D, Candolle G, Campana A. Minilaparotomy and endoscopic techniques for tubal sterilisation. Cochrane Database Syst Rev 2004;(2):CD001328. DOI:10.1002/14651858.CD001328.pub2
2. Royal College of Obstetricians and Gynaecologists. Male and Female Sterilisation: Evidence-based esearch-services/ -and-female-sterilisation/ (accessed 13 April 2014)

3. Huber AW, Mueller MD, Ghezzi F, et al. Tubal sterilization: Complications of laparoscopy and minilaparotomy. Eur J Obstet Gynecol Reprod Biol 2007;134(1):105-109. DOI:10.1016/j. ejogrb.2006.06.0164

4. South Africa Demographics Profile 2014. http://www.indexmundi.com/south_africa/ demographics profile.html (accessed 8 April 2015).

5. United Nations Population Division. Meeting Demand for Family Planning. 2015:1-2. http:// www.un.org/en/development/desa/population/publications/pdf/popfacts/popfacts_2013-6.pdf (accessed 8 April 2015)

6. Weigel R, Estill J, Egger M, et al. Mortality and loss to follow-up in the first year of ART: Malaw national ART programme. AIDS 2012;26(3):365-373. DOI:10.1097/QAD.0b013e32834ed814

7. Mollentze WF. Obesity in South Africa: A call for action. J Endocrinol Metab Diabetes S Af 2006;11(2): 44-45.

8. Stephenson R, Beke A, Tshibangu D. Community and Health Facility Influences on Contraceptive Method Choice in the Eastern Cape, South Africa. WP-07-99. MEASURE Evaluation. 2012:919 966. https://www.guttmacher.org/about/journals/ipsrh/2008/06/community-and-health-facilityinfluences-contraceptive-method-choice (accessed 1 May 2013).

9. Zite N, Borrero S. Female sterilisation in the United States. Eur J Contracept Reprod Health Care 2011;16(5):336-340. DOI:10.3109/13625187.2011.604451

10. Potter JE, Perpétuo IHO, Berquó E, et al. Frustrated demand for postpartum female sterilization in Brazil. Contraception 2003;67(5):385-390. DOI:10.1016/S0010-7824(03)00039-8

11. Essack Z, Strode A. "I feel like half a woman all the time": The impacts of coerced and forced sterilisations on HIV-positive women in South Africa. Agenda Empower Women Gend Equity 2012;26(2):24-34. DOI:10.1080/10130950.2012.708583

12. Garcia-Padial J, Banfield MA, Parker WH. Laparoscopic postpartum tubal sterilization in the morbidly obese patient. J Gynecol Surg 2004;20:89-92. DOI:10.1089/gyn.2004.20.89

13. Chi IC, Mumford SD, Gardner SD. Pregnancy risk following laparoscopic sterilization in nongravid and gravid women. J Reprod Med 1981;26(6):289-294.

14. Chi I-C, Laufe LE, Gardner SD, Tolbert MA. An epidemiologic study of risk factors associated with pregnancy following female sterilization. Am J Obstet Gynecol 1980;136(6):768-773. DOI:10.5555/ uri:pii:0002937880904548

15. Peterson HB, Xia Z, Hughes JM, et al. The risk of pregnancy after tubal sterilization: Finding from the US Collaborative Review of Sterilization. Am J Obstet Gynecol 1996:174(4):1161-1170.

16. Trussell J, Guilbert E, Hedley A. Sterilization failure, sterilization reversal, and pregnancy after sterilization reversal in Quebec. Obstet Gynecol 2003;101(4):677-684.

17. Madari S, Varma R, Gupta J. A comparison of the modified Pomeroy tubal ligation and Filshie clips for immediate postpartum sterilisation: A systematic review. Eur J Contracept Reprod Health Care 2011;16(5):341-349. DOI:10.3109/13625187.2011.594919

18. Varma R, Gupta JK. Failed sterilisation: Evidence-based review and medico-lega ramifications. BJOG Int J Obstet Gynaecol 2004;111(1):1322-1132. DOI:10.1111/j.14710528.2004.00281.x 